\title{
Blind Estimation of Timing Errors in Interleaved AD Converters
}

\author{
Jonas Elbornsson, Jan-Erik Eklund \\ Division of Communications Systems Department of Electrical \\ Engineering \\ Linköpings universitet, SE-581 83 Linköping, Sweden \\ WWW: http://wWw.comsys.isy.liu.se \\ Email: jonas@isy.liu.se, @isy.liu.se
}

November 8, 2000

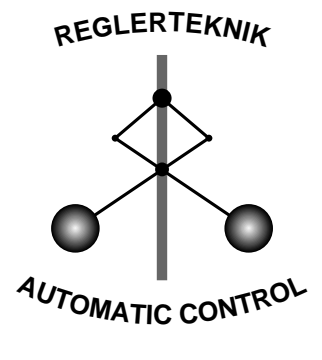

LINKÖPING

Report No.: LiTH-ISY-R-2315

Submitted to ICASSP2001, Salt Lake City, UT, USA

Technical reports from the Communications Systems group in Linköping are available by anonymous ftp at the address ftp.control.isy.liu.se. This report is contained in the file 2315.pdf. 


\begin{abstract}
Parallel AD converter structures is one way to increase the sampling rate. Instead of increasing the sample rate in one $\mathrm{AD}$ converter, several AD converters with lower sampling rate can be used instead. A problem in these structures is that the time between samples is usually not equal because there are errors in the delays between the AD converters. We will here present a method to estimate the timing offset errors. The estimation algorithm works without any special calibration signal, instead the normal input signal is used. The only assumption that we need on the input signal is that most of the energy is concentrated to a low pass band, below about $1 / 3$ of the Nyquist frequency. Simulations of the time interleaved AD converter show that the method estimates the errors with high accuracy.
\end{abstract}

Keywords: non-uniform sampling, AD conversion, interleaved, blind estimation 


\section{BLIND ESTIMATION OF TIMING ERRORS IN INTERLEAVED AD CONVERTERS}

\author{
J. Elbornsson \\ Linköpings universitet \\ Department of Electrical Engineering \\ SE-581 83 Linköping,SWEDEN \\ jonas@isy.liu.se
}

\author{
J.-E. Eklund \\ Ericsson Microelectronics AB \\ SE-581 17 Linköping, SWEDEN \\ jan-erik.eklund@mic.ericsson.se
}

\begin{abstract}
Parallel AD converter structures is one way to increase the sampling rate. Instead of increasing the sample rate in one AD converter, several $\mathrm{AD}$ converters with lower sampling rate can be used instead. A problem in these structures is that the time between samples is usually not equal because there are errors in the delays between the AD converters. We will here present a method to estimate the timing offset errors. The estimation algorithm works without any special calibration signal, instead the normal input signal is used. The only assumption that we need on the input signal is that most of the energy is concentrated to a low pass band, below about $1 / 3$ of the Nyquist frequency. Simulations of the time interleaved AD converter show that the method estimates the errors with high accuracy.
\end{abstract}

\section{INTRODUCTION}

Many digital signal processing applications, such as radio base stations or VDSL modems, require AD converters with very high sample rate. To achieve high enough sample rates, an array of $\mathrm{M}$ $\mathrm{AD}$ converters in parallel can be used. Each ADC should work at $1 / M$ th of the desired sample rate. To avoid time drift between the different ADC's, the same master clock is used for all ADCs, see figure 1 . To get the same sample interval for all samples the

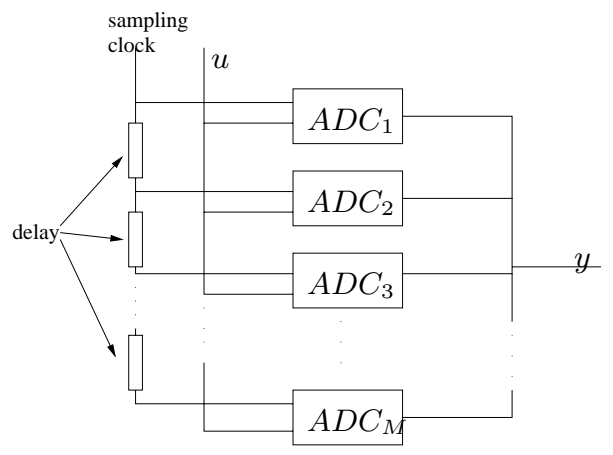

Fig. 1. M parallel ADC's with the same master clock.

$n$th ADC should be delayed $\frac{n T_{s}}{M} s$, where $T_{s}$ is the sample interval for the master sampling clock [1]. If there are errors in the delay times we will get a non-uniformly sampled signal. If these errors are not compensated for, the resolution of the ADC will decrease with several bits $[2,3,4]$. However, if the delay errors are known, the true signal can be recovered $[5,6]$. The errors are assumed to be static, so that the error is the same in the same AD-converter from one cycle to the next. There are also random errors in time and amplitude due to thermal noise, that are different from one sample to the next. These errors do not have anything to do with the parallel structure of the AD-converter and are not discussed further here. There are some presented methods to estimate timing errors in interleaved AD converters, see [7, 8]. But these methods assume a known input signal. Here we will present a method for estimation of these errors with unknown input signal.

\section{NOTATION}

The analog input signal is denoted $u(t) . T_{s}$ denotes the nominal sampling time, that we would have without any errors. $M$ is the number of $\mathrm{AD}$ converters in the parallel structure. The time offset for the $i$ th $\mathrm{AD}$ converter is denoted $t_{i}$. The output from the $i$ th $\mathrm{AD}$ converter is denoted $y_{i}[k]$ where $k$ is the $k$ th sample from that $\mathrm{AD}$ converter. Each $\mathrm{AD}$ converter form a subsequence, $y_{i}[k]=u\left((k M+i) T_{s}+t_{i}\right)$. The sample time for each such subsequence is exactly $M T_{s}$. These subsequences are merged to the output signal $y[m]=y_{(\operatorname{mmod} M)}\left[\left\lfloor\frac{m}{M}\right\rfloor\right]$, where $\lfloor\cdot\rfloor$ denotes integer part. The difference between samples from AD converter $i-1$ and $\mathrm{AD}$ converter $i$ is denoted $\Delta y_{i}[k]=y_{i}[k]-y_{i-1}[k]$. We denote by $N$ the number of data points from each $\mathrm{AD}$ converter. We assume that the same number of samples is taken from all the AD converters so that $N M$ is the total number of data. We use the notation $\bar{E}(s(t))=\lim _{n \rightarrow \infty} \frac{1}{n} \sum_{t=1}^{n} E(s(t))$ for quasistationary signals [9], where the expectation is taken over possible stochastic parts of $s(t)$.

\section{ESTIMATION METHOD}

Throughout the report we will assume that the AD-converters are infinite precision sampling units, i.e. we have no amplitude quantization. We also assume that amplitude errors are zero.

\subsection{Timing offset estimation}

For the timing offset estimation algorithm we need a bandwidth assumption on the input signal, it must have most of its energy in a frequency band below about $1 / 3$ of the Nyquist frequency. This restriction is not limiting the use of the algorithm since we need an anti-aliasing filter before the AD converter anyway. The algorithm is based on the assumption that the signal changes more on average if it is a long time between the samples than if it is a short time between them. Therefore we have to assume that the signal varies slow enough, i.e. has low enough bandwidth. We look at the difference, $\Delta y_{i}[k]$, between two adjacent samples and make a Taylor expansion around the nominal sampling time of AD converter $i-1$.

$$
\begin{aligned}
\Delta y_{i}[k] & =y_{i}[k]-y_{i-1}[k] \\
& \approx\left(T_{s}+t_{i}-t_{i-1}\right) u^{\prime}\left(k M T_{s}+(i-1) T_{s}\right)
\end{aligned}
$$


We calculate the mean squared difference between two adjacent AD converters:

$$
\begin{aligned}
\hat{R}_{i, i-1}^{N}[0] & =\frac{1}{N} \sum_{k=1}^{N}\left\{\Delta y_{i}[k]\right\}^{2} \\
& \rightarrow\left(T_{s}+t_{i}-t_{i-1}\right)^{2} \bar{E}\left\{\left(u^{\prime}(t)\right)^{2}\right\}, N \rightarrow \infty
\end{aligned}
$$

If the mean value of the input signal derivative is known we can calculate the time offsets from equation (2) using the first time offset as reference, i.e. assuming $t_{1}=0$ :

$$
t_{i}=\sum_{j=2}^{i}\left(\sqrt{\frac{\hat{R}_{j, j-1}^{N}[0]}{E\left\{\left(u^{\prime}(t)\right)^{2}\right\}}}-T_{s}\right), i=2, \ldots, M
$$

Since the mean value of $\left(u^{\prime}(t)\right)^{2}$ is usually unknown we have to estimate it from the sampled data. $E\left(u^{\prime}(t)\right)^{2}$ can be estimated as an average over all the AD-converters:

$$
\begin{aligned}
& \frac{1}{M} \sum_{i=1}^{M} \hat{R}_{i, i-1}^{N}[0]=\frac{1}{N M} \sum_{k=1}^{N} \sum_{i=1}^{M}\left(y_{i}[k]-y_{i-1}[k]\right)^{2} \\
\approx & \frac{1}{N M} \sum_{k=1}^{N} \sum_{i=1}^{M}\left(T_{s}+t_{i}-t_{i-1}\right)^{2}\left(u^{\prime}\left(k M T_{s}+(i-1) T_{s}\right)\right)^{2} \\
\rightarrow & \frac{1}{M} \sum_{i=1}^{M}\left(T_{s}+t_{i}-t_{i-1}\right)^{2} E\left\{\left(u^{\prime}(t)\right)^{2}\right\} \\
= & \left(T_{s}^{2}+\frac{2}{M} \sum_{i=1}^{M} t_{i}^{2}-\frac{2}{M} \sum_{i=1}^{M} t_{i} t_{i-1}\right) E\left\{\left(u^{\prime}(t)\right)^{2}\right\}
\end{aligned}
$$

From equation (4) it seems like we have to know the time offset to calculate the estimate of $E\left\{\left(u^{\prime}(t)\right)^{2}\right\}$. We can however get a crude estimate by assuming that $\frac{2}{M} \sum_{i=1}^{M} t_{i}^{2}-\frac{2}{M} \sum_{i=1}^{M} t_{i} t_{i-1}$ is small. This is a quite reasonable assumption if the timing offset errors are small on average compared to the nominal sampling time. Using this estimate we can calculate a crude estimate of the time offsets using equation (3):

$$
t_{i}^{(0)}=\sum_{j=2}^{i}\left(\sqrt{\frac{\hat{R}_{j, j-1}^{N}[0]}{\frac{1}{M T_{s}^{2}} \sum_{i=1}^{M} \hat{R}_{i, i-1}^{N}[0]}}-T_{s}\right), i=2, \ldots, M
$$

With this estimate of the time offsets we can improve the estimate of $E\left\{\left(u^{\prime}(t)\right)^{2}\right\}$ using equation (4). Then the time offset estimates can be improved by fixed-point iteration [10]:

$$
\begin{gathered}
t_{i}^{(l)}=\sum_{j=2}^{i}\left(\sqrt{\frac{\hat{R}_{j, j-1}^{N}[0]}{\frac{1}{a^{(l-1)}} \sum_{i=1}^{M} \hat{R}_{i, i-1}^{N}[0]}}-T_{s}\right), i=2, \ldots, M \\
\text { where } a^{(l)}=M\left(T_{s}^{2}+\frac{2}{M} \sum_{i=1}^{M}\left(t_{i}^{(l)}\right)^{2}-\frac{2}{M} \sum_{i=1}^{M} t_{i}^{(l)} t_{i-1}^{(l)}\right)
\end{gathered}
$$

\subsection{Noise sensitivity}

The algorithm in the previous section is based on the assumption that adjacent samples are correlated, i.e. that the signal is bandlimited. We will in this section discuss how the estimation is affected by an additive noise term at the output of the AD converter:

$$
y_{i}[k]=u\left((k M+i) T_{s}+t_{i}\right)+e_{i}[k], e_{i}[k] \in N(0, \lambda)
$$

From equation (1) we now have

$$
\begin{aligned}
\Delta y_{i}[k] & \approx\left(T_{s}+t_{i}-t_{i-1}\right) u^{\prime}\left(k M T_{s}+(i-1) T_{s}\right) \\
& +e_{i}[k]-e_{i-1}[k]
\end{aligned}
$$

Since we assume that the noise is independent of $u(t)$ and the different noise sources are independent of each other the cross terms disappear and we have from equation (8):

$$
\begin{aligned}
\hat{R}_{i, i-1}^{N} & =\frac{1}{N} \sum_{k=1}^{N}\left\{\Delta y_{i}[k]\right\}^{2} \\
& \rightarrow\left(T_{s}+t_{i}-t_{i-1}\right)^{2} E\left\{\left(u^{\prime}(t)\right)^{2}\right\}+2 \lambda, N \rightarrow \infty
\end{aligned}
$$

With (9) in (7) we can see that the noise will cause an error in the time error estimates:

$$
\begin{aligned}
t_{i}^{(l)} & =\sum_{j=2}^{i}\left(\sqrt{\frac{\left(\hat{R}_{j, j-1}^{N}\right)_{y^{0}}+2 \lambda}{\frac{1}{a^{(l-1)}} \sum_{i=1}^{M}\left(\hat{R}_{i, i-1}^{N}\right)_{y^{0}}+\frac{M}{a^{(l-1)}} 2 \lambda}}-T_{s}\right) \\
i & =2, \ldots, M \\
a^{(l)} & =M\left(T_{s}^{2}+\frac{2}{M} \sum_{i=1}^{M}\left(t_{i}^{(l)}\right)^{2}-\frac{2}{M} \sum_{i=1}^{M} t_{i}^{(l)} t_{i-1}^{(l)}\right)
\end{aligned}
$$

Here $\left(\hat{R}_{i, i-1}^{N}\right)_{y^{0}}=\frac{1}{N} \sum_{k=1}^{N}\left\{\Delta y_{i}^{0}[k]\right\}^{2}$, where $y_{i}^{0}[k]$ denotes the noise-free part of $y_{i}[k]$. From the equation above we can see that the estimates are more accurate when the signal is varying quickly (the derivatives get larger). The accuracy of the first order Taylor expansion approximation decreases however when the signal is fast varying. This means that if we can choose the frequency content in the input signal there is a trade-off between accuracy of the Taylor approximation and accuracy of the time estimation algorithm in the noise corrupted case. If the noise variance, $\lambda$, is known, we can compensate for the noise in the algorithm by subtracting $2 \lambda$ in equation (9).

\subsection{Estimation of the noise variance}

As we saw in the previous section, the performance of the estimation algorithm can be quite bad if there is a lot of noise present in the signals. To improve the performance of the timing estimation when we have noisy measurements, we have to estimate the noise variance, $\lambda$, since it is usually unknown. With this algorithm we can also handle the case where parts of the signal lies in the frequency band above $1 / 3$ of the Nyquist frequncy, since this part can be described by the noise term. The measurement from each ADC can be described as a regression of old measurements plus some noise:

$$
y_{i}[k]=\sum_{j=1}^{l} a_{i, j} y[(k-1) M+i-j]+e_{i}[k], i=1, \ldots, M
$$

This model can be used to predict the signal part of the next measurement:

$$
\hat{y}_{i}^{0}[k]=\sum_{j=1}^{l} a_{i, j} y[(k-1) M+i-j], i=1, \ldots, M
$$

The $a_{i, j}$ coefficients can be estimated from data by minimizing a loss function, the mean square error:

$$
\begin{array}{r}
V_{i}\left(a_{i, j}, j=1, \ldots, l\right)=\sum_{k=1}^{N}\left(\hat{y}_{i}[k]-y_{i}[k]\right)^{2} \\
\left(\hat{a}_{i, j}, j=1, \ldots, l\right)=\operatorname{argmin}_{i}\left(a_{i, j}, j=1, \ldots, l\right)
\end{array}
$$




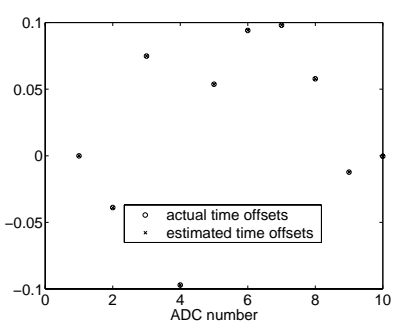

Fig. 2. Timing offset estimation, sinusoidal input with frequency $\omega=0.02 \mathrm{rad} / \mathrm{s}$.

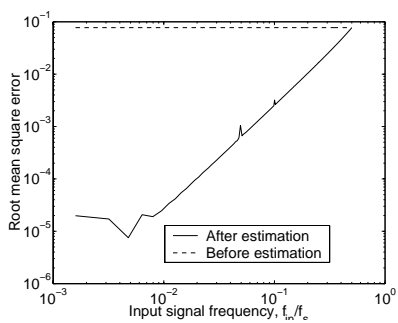

Fig. 3. Timing offset estimation, sinusoidal input with frequency up to the Nyquist frequency.

From the model 11 we can see that

$$
\begin{array}{r}
V_{i}\left(a_{i, j}^{*}, j=1, \ldots, l\right)=\sum_{k=1}^{N}\left(y_{i}^{0}[k]-y_{i}[k]\right)^{2} \\
=\sum_{k=1}^{N}\left(e_{i}[k]\right)^{2}=\lambda
\end{array}
$$

where $a_{i, j}^{*}$ are the true parameters, that $\hat{a}_{i, j}$ is an estimate of. This means that the noise variance, $\lambda$, can be estimated from data before the timing error estimation and the estimated value can be subtracted as described in the previous section.

\section{SIMULATIONS}

Throughout this section we have simulated 10 AD-converters in parallel if nothing else is mentioned. We have here assumed that the AD-converters are perfect samplers so there are no other errors than timing errors. The precision is assumed to be infinite. The sample interval is set to one since any other sample interval is just a scaling under the conditions assumed above.

\subsection{The timing offset estimation}

In the simulations of $\mathrm{AD}$-converters with timing offsets we have used random timing offsets between $-10 \%$ and $10 \%$ of the nominal sampling time. We have used 100000 samples in the simulations if nothing else is mentioned. The nominal sampling frequency is $f_{s}=1 \mathrm{~Hz}$ in all the simulations. Figure 2 shows the result of the timing offset estimation when a sinusoidal input with frequency $\omega=0.02 \mathrm{rad} / \mathrm{s}$ has been used. The estimation algorithm works best for low frequencies, but the errors stay below $1 \%$ for frequencies up to about $1 / 3$ of the Nyquist frequency. In Figure 3 the root mean square error is plotted after estimation with sinusoidal input signals with frequencies up to the Nyquist frequency. If there is additive white noise on the signal the estimation accuracy decreases, especially for low frequency signals. That is because the derivative of a low frequency signal is small compared to the noise, see Figure 4. The estimation algorithm has

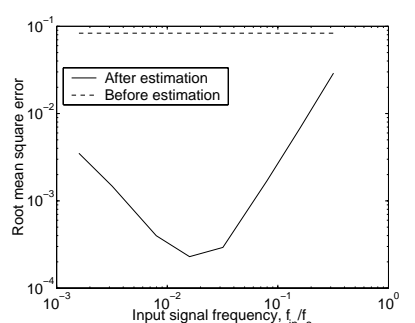

Fig. 4. Timing offset estimation error, sinusoidal with noise, $S N R=60 d B$.

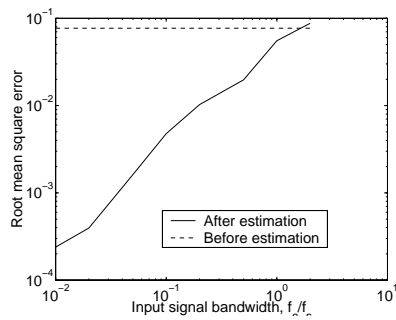

Fig. 5. Timing offset estimation error, band-limited white noise with different bandwidths as input signals.

also been tested with other signals, for example sums of sinusoids and band-limited white noise. In Figure 5 the estimation error is shown for estimation from band-limited white noise with different bandwidths. From all these simulations we can see that the estimation algorithm works well for most kinds of signals. If the estimates are updated slowly, e.g. with a forgetting factor close to one, "bad" parts of the signal will not destroy the estimation accuracy. Simulations have also shown that accuracy of the estimates is significantly improved with more data up to about $10^{6}$ samples, especially for noisy signals. With more than $10^{6}$ samples the improvement is quite small however, see Figure 6 . We can also see that the iteration in equation 6 is quite important. One iteration is, however, usually enough, see Figure 7.

\subsection{Timing offset estimation with estimated noise variance}

In this section we have simulated errors between $-50 \%$ and $50 \%$ of the nominal sampling time to see the effects of the noise estimation more clearly. In Figure 8 timing estimation without compensation for $\lambda$ is compared with timing estimation with compensation for the true $\lambda$ and compensation for the estimated $\lambda$. The estimated $\lambda$ has been estimated with a regression of 200 measurements backwards in time. We can see here that compensation with estimated $\lambda$ gives almost as good timing error estimation as compensation with the true $\lambda$. Figure 9 shows how estimation accuracy depends on the length of the regression vector. We can see here that about

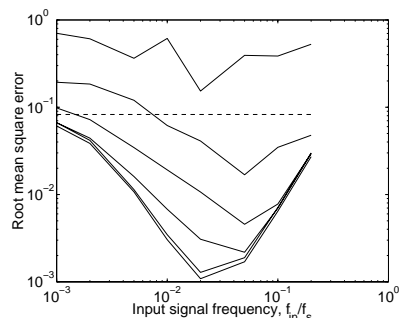

Fig. 6. timing offset estimation errors for different number of data, between 10 and 1000000 . 

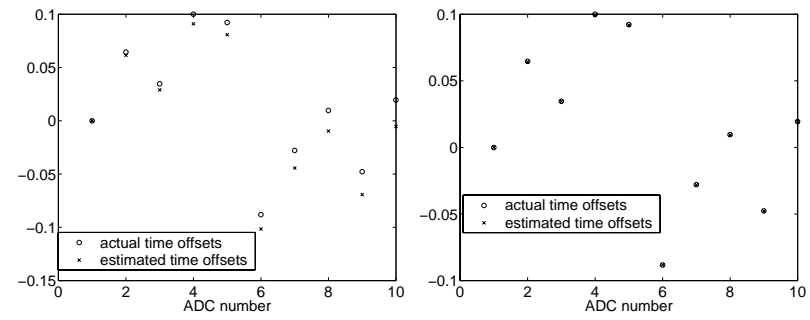

Fig. 7. To the left: estimation error before iteration. To the right: estimation error after one iteration.

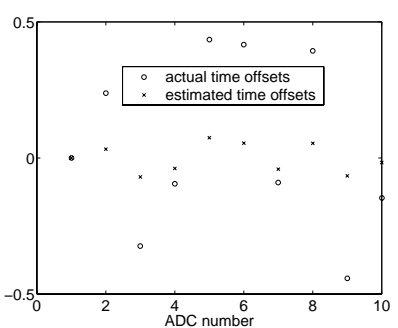

(a) timing estimation without compensation for $\lambda$

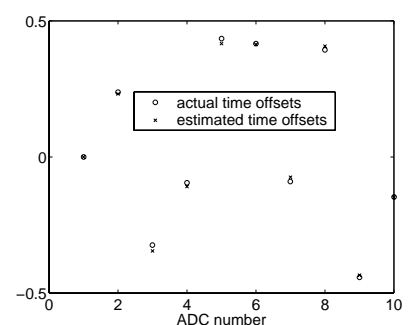

(b) compensation with true $\lambda$

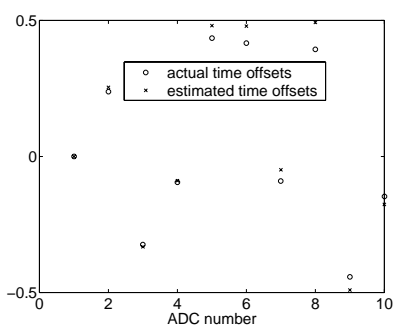

(c) compensation with estimated $\lambda$

Fig. 8. Comparison of estimation accuracy with and without compensation for $\lambda$

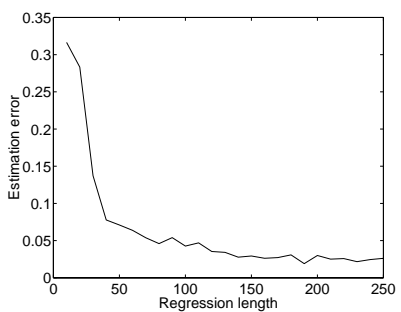

Fig. 9. Estimation accuracy dependence on regression length
150 samples in the regression vector is needed to get a good estimation of $\lambda$.

\section{CONCLUSIONS}

Parallel AD-converters are used for increasing the sample rate by letting each $\mathrm{AD}$-converter work at a lower sampling rate. A problem in these $\mathrm{AD}$-converter structures is the synchronization between the AD-converters. We have presented a method to estimate timing offsets in a parallel AD-converter structure. The estimation works without knowledge of the input signal. The only assumption that is needed about the signal is that the energy spectrum should be concentrated to the lower frequencies for the timing offset estimation to work. The timing offset estimation works well for signals band-limited to about $1 / 3$ of the Nyquist frequency. The accuracy is however still improved even if the bandwidth is at the Nyquist frequency. Noisy measurements can be handled by compensating for the noise variance in the algorithm. We have also presented a method to estimate the noise variance before estimating the timing errors.

\section{REFERENCES}

[1] Y-C Jenq, "Digital spectra of nonuniformly sampled signals: A robust sampling time offset estimation algorithm for ultra high-speed waveform digitizers using interleaving," IEEE Transactions on Instrumentation and Measurement, vol. 39, no. 1, pp. 71-75, February 1990.

[2] S.K. Mitra A. Petraglia, "Analysis of mismatch effects among A/D converters in a time-interleaved waveform digitizer," IEEE Transactions on Instrumentation and Measurement, vol. 40, no. 5, pp. 831-835, October 1991.

[3] Y-C Jenq, "Digital spectra of nonuniformly sampled signals: Fundamentals and high-speed waveform digitizers," IEEE Transactions on Instrumentation and Measurement, vol. 37, no. 2, pp. 245-251, June 1988.

[4] Y-C Jenq, "Digital spectra of nonuniformly sampled signals: Digital look-up tunable sinusoidal oscillators," IEEE Transactions on Instrumentation and Measurement, vol. 37, no. 3, pp. 358-362, September 1988.

[5] L. Cuvelier L. Vandendorpe, B. Maison, "RLS design of polyphase components for the interpolation of periodically noonuniformly sampled signals," in International Conference on Acoustics, Speech, and Signal Processing, 1995. IEEE, 1995, vol. 2, pp. 1229-1232.

[6] M. Ganshadzahi F. Marvasti, M. Analoui, "Recovery of signals from nonuniform samples using iterative methods," IEEE Transactions on Signal Processing, vol. 39, no. 4, pp. 872-878, April 1991.

[7] F.H. Irons D.M. Hummels, J.J. McDonald II, "Distortion compensation for time-interleaved analog to digital converters," in IEEE Instrumentation an Measurement Technology Conference, 1996. IEEE, June 1996, pp. 728-731.

[8] J.J. Corcoran, "Timing and amplitude error estimation for time-interleaved analog-to-digital converters," US Patent nr. 5,294,926, October 1992

[9] L. Ljung, System Identification, Theory for the user, Prentice-Hall, 2 edition, 1999.

[10] G. Dahlquist Å. Björk, "Numerical mathematics,” July 1997. 